\title{
THE USE OF THE PHONOCARDIOGRAPH IN CLINICAL CARDIOLOGY
}

\author{
September 11, 1947. Section of Cardiology
}

\section{By William Evans, London}

The phonocardiograph was born soon after the electrocardiograph, but their growth has been unequal in that the latter has been applied immediately to clinical medicine and has flourished while the former test, used to display such murmurs as the presystolic and the one identified with patent ductus arteriosus, has become largely exhibitional and for that reason has lost ground. More recently, however, phonocardiography has shown signs of coming into its own, and he now predicted for it a place in cardiology not far behind electrocardiography. He proposed to show some of the ways in which the test had already contributed to our knowledge and to point the way in which it could be usefully developed in the future.

In the first place, it had confirmed the clinical classification for Triple Heart Rhythm. Such a classification had become necessary owing to the confused terminology connected with this rhythm. All cases of triple heart rhythm fall naturally into three groups. First, from addition of the third heart sound which immediately follows the second sound; secondly, from addition of the fourth heart sound which immediately precedes the first heart sound, and thirdly, from addition of a sound in late systole. This third variety was an innocent condition and only assumed importance in its differentiation from the presence of the third heart sound.

The first kind of triple heart rhythm was found in healthy subjects and in those conditions that produce right heart failure: this included mitral stenosis, hypertensive heart failure, emphysema, pulmonary embolism, primary pulmonary hypertension, auricular septal defect, constrictive pericarditis, cardiac infarction, and anæmia. He spoke briefly about each of these forms of triple heart rhythm.

The second variety from addition of the fourth heart sound was found in prolonged $A-V$ conduction or in left ventricular heart failure as in hypertensive heart failure, aortic incompetence and failure, and in cardiac infarction.

Turning to Heart Murmurs, he stressed the importance of the phonozardiograph in confirming the reliability of certain clinical signs which had been assembled for the differentiation of innocent heart murmurs into five main varieties, and he outlined such signs. Concerning the murmurs of organic heart disease he mentioned how common it was for the phonocardiograph to show that the systolic murmur of mitral disease actually occurred during auricular systole as in the case of the presystolic murmur. The mid-diastolic murmur of mitral stenosis was even more important in the light of this newer test, so that in 74 cases of undoubted mitral stenosis the test had shown the presence of this murmur in all of them, but more cases need to be tested before one could say that it was an invariable finding. He then dealt with the murmurs of aortic valvular disease, hypertension, congenital heart disease, anæmia, and heart block.

Dr. Evans believed in the importance of the test not only for research purposes but also for clinical diagnosis in specific instances. The test had come to stay. We would not be able to live without it and for the good reason that our patients would not be able to live so well without it.

In conclusion, he stressed three things in clinical auscultation. First, the advantage to clinical diagnosis of finding and placing the added heart sound which created a triple heart rhythm. Secondly, that the quality and intensity of murmurs matter far less than their place in the cardiac cycle and in relation to the heart sounds. Thirdly, and above all else, the phonocardiograph had emphasized the importance of self catechism during clinical auscultation. He mentioned six questions which should be applied when the bell of the stethoscope rested on any part of the chest in the search for heart disease.

\section{By Camille Lian, Paris}

I appreciate the honour of being invited to attend this International Conference and to address the Section of Cardiology. I have been specially interested in phonocardiography for many years and, together with a distinguished qualified engineer, Mr. Minot, have succeeded in designing an original 
phonocardiograph, known in France as the LianMinot Telestethophone. With this apparatus it is possible to record the heart sounds while actually hearing them reproduced by loud-speaker, a feature of capital importance. The electrocardiogram and the apex impulse can be recorded simultaneously with the heart sounds.

I propose to speak to-day about triple rhythm which I regard as the principal indication for employing phonocardiography in the clinic.

I shall first consider that variety of triple rhythm in which the second sound is single, and where we have to distinguish between gallop rhythm and a reduplication of the first heart sound (Fig. 1 and 2). The clinical diagnosis is usually easy-in gallop rhythm, the extra sound is separated from the first heart sound by a silent interval, but there is no silent interval between the two constituents of a reduplicated first sound. However, I have established by phonocardiography that, exceptionally, this silent interval between the gallop sound and the first sound is lacking, and I have described this particular phenomenon as delayed presystolic gallop (galop présystolique retardé) (Fig. 3). In this case, the clinician is likely to make the serious error of mistaking a gallop rhythm, which signifies left ventricular failure, for a reduplicated first sound which is a sign of little importance. Here the phonocardiograph triumphs over the stethoscope. In the phonocardiogram, if the first constituent of the double sound precedes the summit of the $R$ wave of the electrocardiogram, then we are dealing with a presystolic gallop. If, on the other hand, both constituents of the double sound fall after the summit of the $\mathbf{R}$ wave, then we are dealing with a reduplication of the first heart sound.

These remarks apply to records obtained by the Lian-Minot phonocardiograph which is not sensitive to vibrations of low frequency, for its curve of sensitivity is almost identical with that of the human ear. For this reason, in my phonocardiograms of healthy subjects, no part of the vibrations belonging to the first sound occurs before the summit of the $R$ wave of the electrocardiogram. Recently, in the United States, Rappaport and Sprague have sup- . ported the view that a phonocardiograph should have the same curve of sensitivity as that of the ear. Contrary to the opinion of the Argentine authorities, they consider, as I do, that the vibrations constituting the normal first heart sound never occur earlier than the summit of the $R$ wave of the electrocardiogram. Therefore, both components of a reduplicated first sound are always later than the summit of the $\mathbf{R}$ wave. I have also established, both clinically and by phonocardiograph, that there are arterial reduplications of the first sound, for aortic or pulmonary vibrations of appreciable magnitude are added to the ventricular vibrations which constitute the first sound. In these arterial reduplications there is a protosystolic snap of maximum intensity at the right or left second or third intercostal space.

I now come to the second variety of triple rhythm in which the first sound is single. Here the diagnosis lies mainly between a reduplication of the second sound and the triple rhythm of mitral stenosis. I say this because I have established both clinically and by phonocardiography that the triple rhythm of mitral stenosis is constituted not by a reduplication of the second sound but by the opening snap of the mitral valve (Fig. 4).

Triple rhythm in mitral stenosis may also be due to an initial reinforcement of the diastolic murmur, which is characteristic, or sometimes to a reduplication of the second sound which is not characteristic as it occurs in many other conditions. It is therefore important to distinguish a reduplicated second sound, which is not peculiar to mitral stenosis, from the opening snap of the mitral which is quite characteristic (Fig. 5).

I give the following rules for making this distinction. On auscultation, a reduplicated second sound is best heard in the second or third left intercostal space, and the mitral opening snap in the fourth or fifth left space, or less often near the apex. I have also found that a reduplicated second sound is inaudible immediately above the sternum (namely, in the suprasternal notch), while the opening snap is often audible at this site.

In phonocardiography, I prefer to record the apex impulse electrically, as can be done by the electrokymograph of the Lian-Minot apparatus. In such records, the second constituent of a reduplicated second sound occurs during the descent of the apex curve, between points $C$ and $D$. The mitral opening snap occurs at point $D$, and the initial reinforcement of the diastolic murmur, still later, at point $\mathrm{E}$.

It is not essential to record the apex impulse in order to identify these three varieties of triple rhythm, for it can also be done by measuring the time interval between the second heart sound and the extra sound. In the case of a reduplicated second sound, the extra sound occurs from 0.03 to $0.07 \mathrm{sec}$. after the commencement of the second sound. In the opening snap of the mitral, this interval is 0.07 to $0.11 \mathrm{sec}$., and in the initial reinforcement of the diastolic murmur it is 0.10 to 0.17 sec.

In the diagnosis of mitral triple rhythm, there are several other possible sources of error to be considered. There is the snap I have described as the late systolic pleuro-pericardial snap (Fig. 6) which 
FIG. 1.-Reduplication of the first sound. The two groups of vibrations constituting the first sound both occur after the summit of the $R$ wave in the electrocardiogram.
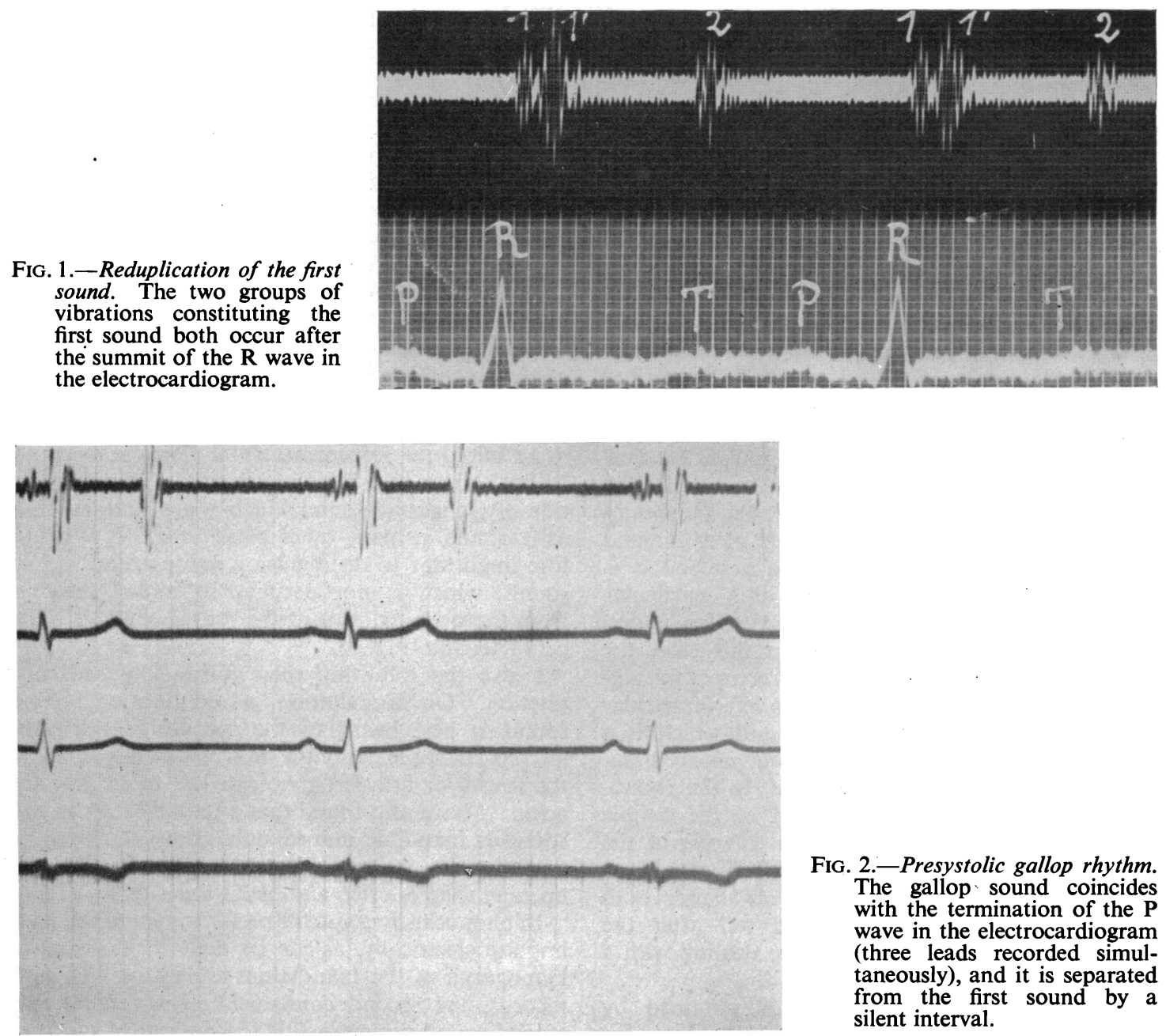

Fig. 2.-Presystolic gallop rhythm. The gallop sound coincides with the termination of the $P$ wave in the electrocardiogram (three leads recorded simultaneously), and it is separated from the first sound by a silent interval.

Fig. 3.--Retarded presystolic gallop rhythm. The gallop sound coincides with the termination of the $P$ wave. There is no silent interval between the gallop sound and the first heart sound.

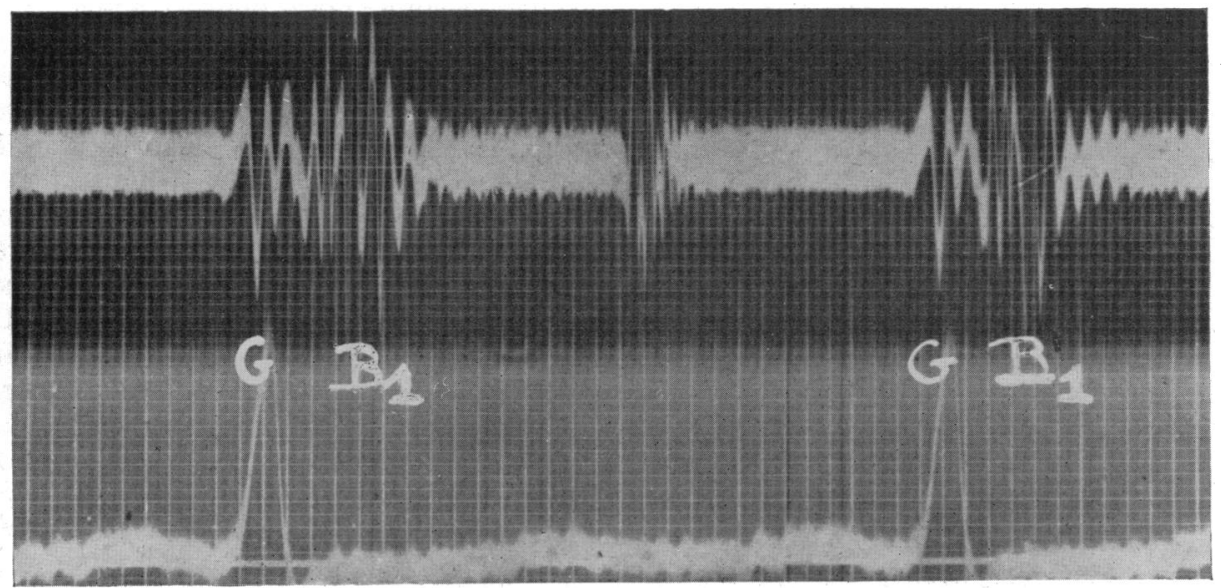




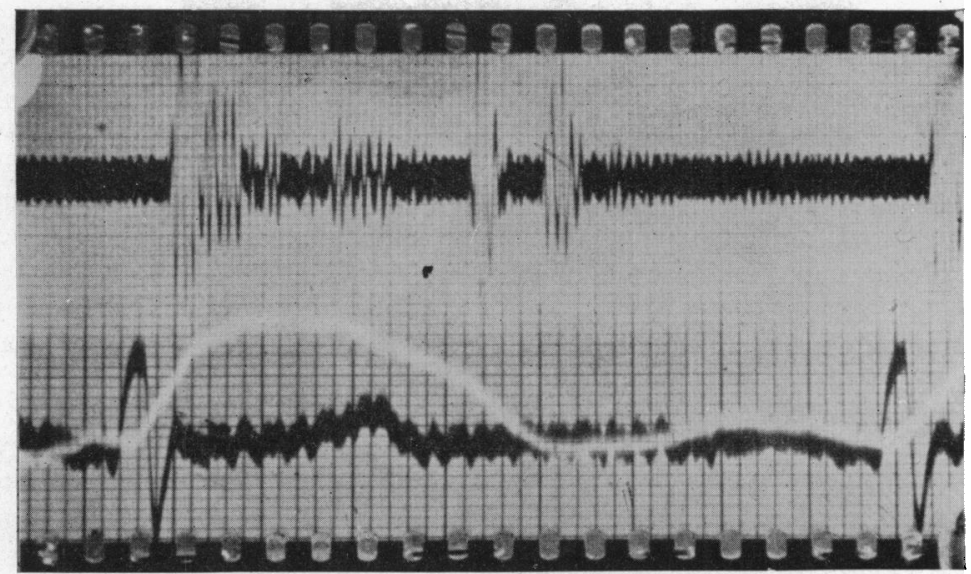

FIG. 4.-Mitral opening snap. Above, the phonocardiogram, below, in white, the apical electromyogram, and in black the electrocardiogram. The record shows, in succession, the first sound, a systolic murmur, the second sound, and finally the opening snap of the mitral. The second sound coincides with the start and the opening snap with the termination of the descending limb of the apical cardiogram. The snap begins 0.07 seconds after the start of the second sound.

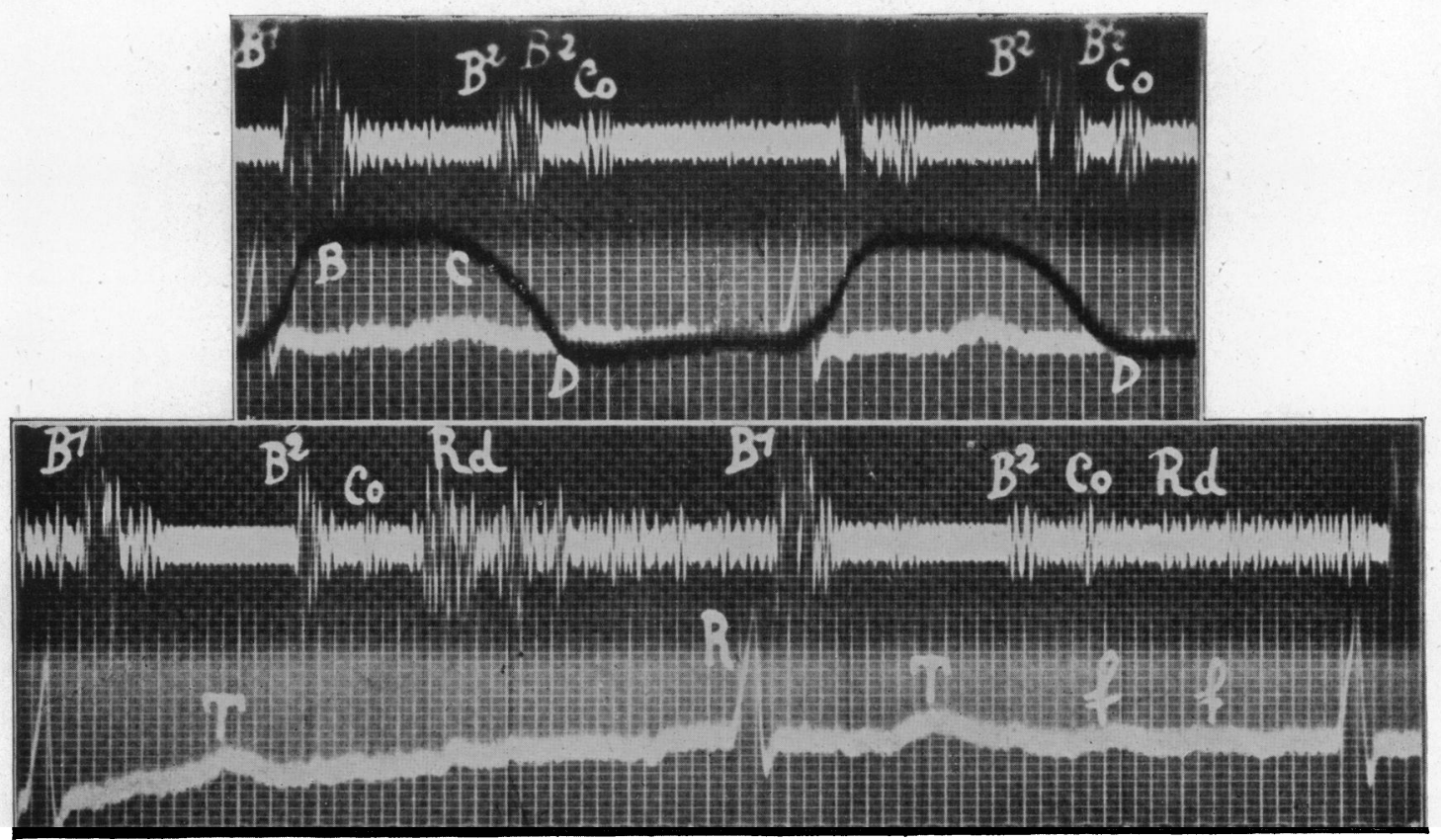

Fig. 5.-The complete phonocardiogram of a case of mitral stenosis.

(A) Above, phonocardiogram taken from the fourth left interspace, and below it, the apical electromyogram in black, and the electrocardiogram in white. These show the two elements of the reduplicated second sound which coincide with the start of the descending line $C D$ of the apical cardiogram, then the mitral opening snap (CO), which coincides with the base of the descending line at $D$.

(B) Below phonocardiogram taken from the apex, in the left lateral decubitus. It shows successively the first sound, the second sound, the snap (CO) which is slight, and the diastolic rumble (Rd) with its initial protodiastolic accentuation. There is no presystolic accentuation owing to the presence of auricular fibrillation.

These two tracings were taken at the same time and from the same patient. 
depends on an adhesion between the left pleura and the pericardium. In 10 per cent of such cases, there is also a late systolic murmur between the snap and the second sound. I have seen many cases in which the diagnosis of mitral stenosis was mistakenly made, simply on account of this late systolic snap, which is of no importance. The clinical recognition of this snap is not very difficult and with the phonocardiograph it is very easy.

The diagnosis between mitral triple rhythm and a protodiastolic gallop (Fig. 7) is not usually difficult as other signs of left ventricular failure accompany the gallop.

More difficult to distinguish from mitral triple rhythm is the physiological third heart sound, often heard in young subjects. It is usually intermittent. If it is permanent, the patient should be instructed to hold his breath after a deep inspiration, when the third heart sound will disappear immediately, a characteristic phenomenon.

Lastly, we must distinguish between mitral triple rhythm and the sign which I have described as the isodiastolic or protodiastolic pericardial snap (Fig. 8) of adhesive pericarditis. This sound is heard at the same site as the mitral opening snap but it is louder. When it is very loud, you may safely make the diagnosis of calcified pericardium.

In conclusion, I hope that I have shown you the great interest that attaches to phonocardiography in the study of triple heart rhythm.

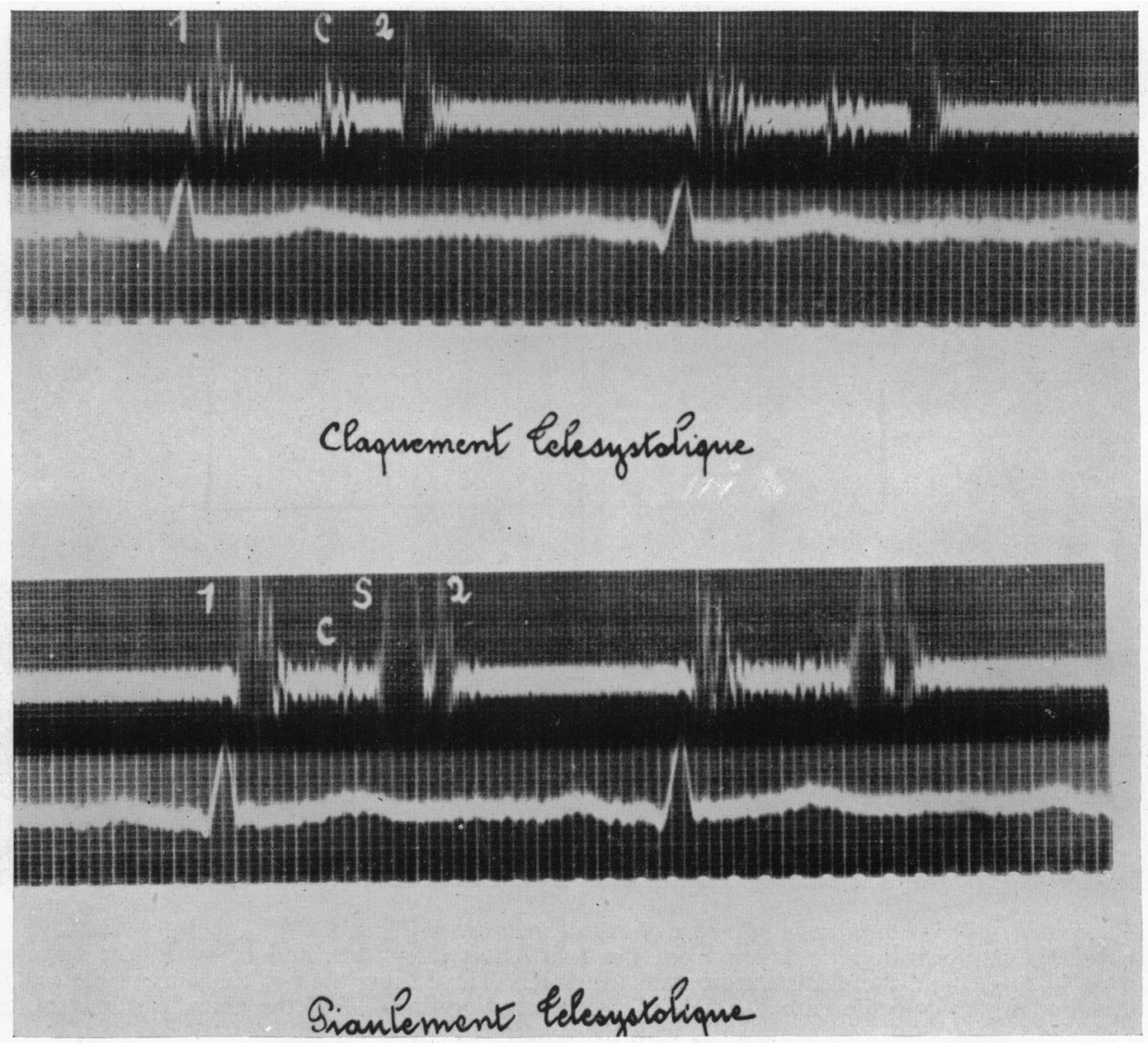

FIG. 6.-Telesystolic snap and murmur due to left-sided pleuro-pericardial adhesions. Above, the snap (C) situated between the first and second sounds, and nearer to the second. Below, the snap (C) is not pronounced, but the late systolic whining murmur is well shown between the snap and the second sound. 


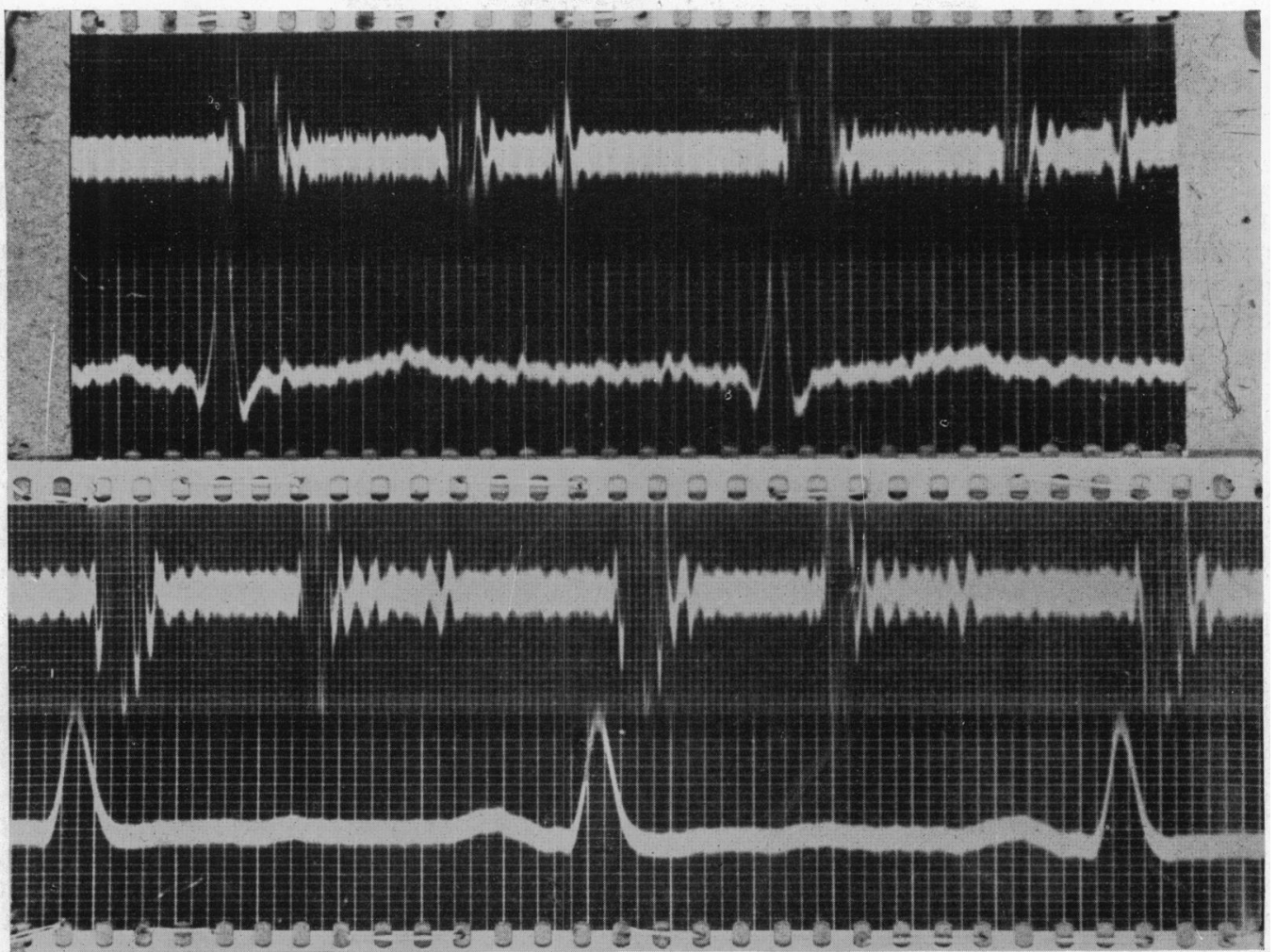

FIG. 7.-Third heart sound and protodiastolic gallop. Above, a third sound begins 0.13 seconds after the onset of the second sound. Below, a protodiastolic gallop sound begins 0.15 seconds after the onset of the second sound. The two records belong to different patients, but the phonocardiographic appearances are similar.

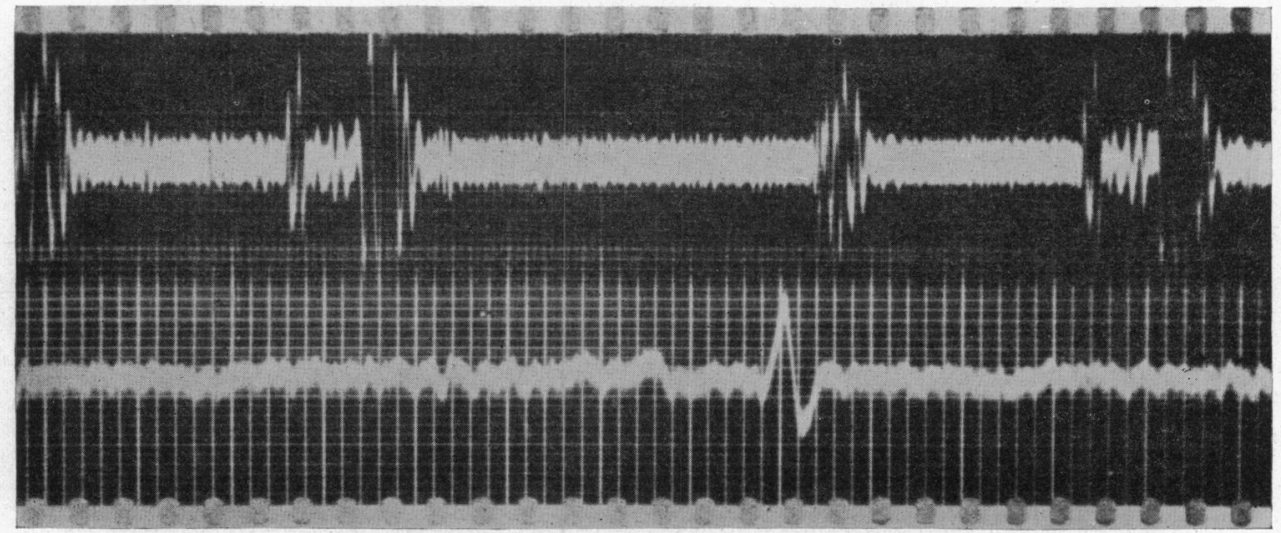

FIG. 8.-Intense isodiastolic pericardial snap (pericardial vibration) in a case of calcified pericardium. The snap occurs 0.08 seconds after the onset of the second sound, and is therefore isodiastolic. The vibrations are of far greater amplitude than those of the second sound. 
Professor Crighton Bramwell (Manchester) said how much he appreciated the privilege of hearing the communication of Professor Lian, an acknowledged master in this subject.

$\mathrm{He}$ then referred to certain difficulties which he himself had encountered in recording the heart sounds. In phonocardiography there were two distinct objectives. One was to obtain a true record of the heart sounds and murmurs, the other to determine their time relation to the events of the cardiac cycle. The second of these was relatively easy, but the first was much more difficult, because the sound picture was apt to be contaminated by instrumental artifacts. Wigger's optical method of recording heart sounds, employing a delicate rubber membrane and a very light mirror, was quite adequate for timing murmurs but did not give a true sound picture. Professor Bramwell showed a phonocardiogram taken from a patient with mitral stenosis and partial heart block. The murmurs in this case differed from cycle to cycle and would have been difficult to interpret without a graphic record. In cycles in which the $\mathbf{P}-\mathbf{R}$ interval was prolonged the presystolic murmur died away before the first heart sound. When the ventricle failed to respond there was a loud murmur but no first sound, and in the cycles in which the P-R interval was normal there was no murmur. Clinically the reason for this was obscure but the record indicated that it was due to the fact that these cycles followed a missed beat. The auricle had already discharged its contents into the ventricle and when it contracted a second time the ventricle was already so full that the blood flow through the mitral orifice was not sufficient to produce a murmur.

He had used this method also for recording arterial sounds during deflation of the armlet in the estimation of blood pressure. These observations had revealed an important artifact, namely that if the pick-up is connected to the recorder by a tube, vibration of the air in the tube will distort the record. As in the case of an organ pipe, the frequency of vibration varies inversely with the length of the tube. This error is apt to contaminate all records in which air conduction is employed.

To obtain a true sound picture electrical conduction should be used since it is instantaneous. Further both pick-up and recorder must have a natural frequency higher than that of the vibrations which one wishes to record.

With this in mind he had used a Matthews' oscillograph in conjunction with a condenser microphone. Both these instruments have a natural period of over 10,000 a second which is well above the frequency of even the higher harmonics of the heart sounds. That this instrument does give a true sound picture is proved by the fact that the records are identical in form with those obtained with a cathode ray oscillograph which has no inertia. This apparatus is excellent for research purposes, but is somewhat temperamental, and it was only by having the collaboration of an expert physicist that he had been able to obtain consistently good results. For routine clinical work we want an instrument that is more fool-proof, like that of Lian and Minot or the Sanborn Stetho-cardiette.

Whereas the timing of heart sounds and murmurs by means of phonocardiography was easy, to obtain a true picture of the sound vibrations was more difficult, since the record was apt to be contaminated by instrumental artifacts. Both pick-up and recorder should have a natural frequency higher than that of the vibrations of the heart sounds and murmurs, and transmission should be by electrical and not by air conduction. For routine clinical work the instrument must be fool-proof. 